\title{
Family Interaction Pattern in Wive's of Alcohol Dependents
}

\author{
Chinnu Sebastian ${ }^{1}$, Suja M.K ${ }^{2}$ \\ ${ }^{1}$ PhD Scholar, ${ }^{2}$ Associate Professor Department of Social Work, Amrita School of Engineering, Coimbatore, \\ Amrita Vishwa Vidyapeetham, India
}

\begin{abstract}
Introduction: Alcoholism is a disease which affects the individual and their family hence it is known as a family disease. Alcoholism reflects on the family bonds and it often leads to the complete breakdown of the entire family. The most affected persons are the wives of alcoholics because of their commonly nature and the intimacy in their relationships. The spouses of alcoholics facing severe domestic, financial and emotional violence which leads major psychological problems like anxiety, depression and poor self-esteem. Family interaction pattern (FIP) is defined as methods by which members of the family relate to each other through various mechanisms binding them in the bond of family.

Aim: This research work has made an attempt to study the Family interaction pattern in wive's of alcohol dependents. This study to measure the level of family interaction pattern and significant factors associated with wive's of alcohol dependents of Kottayam district of Kerala, India. Result: Nearly half of the respondents (37\%) have medium level of Family interaction pattern. 33\% have high family interaction pattern and 30\% have low level of family interaction pattern. The study reveals that FIP Score has significant difference with the variables like age, area of residence, education and duration of drinking habit in spouses. The post hoc test indicates that respondents between the age group of 31-40 years, rural area of residence, educated below $10^{\text {th }}$ standard and above 10 years of drinking habit in spouses have better FIP. Religion, type of family, occupation and family monthly income has no significance. Conclusion: This study inspires in realizing the mental, social, marital and family relation among wive's of alcoholics and it will be a great help in promoting family counselling programmes in de- addiction centres.
\end{abstract}

Key words: Alcoholism, Alcohol dependents, Wives, Family interaction pattern.

\section{Introduction}

Alcoholism is one of the major health and social problems around the world, which results in 3.3 million deaths in every year (WHO, 2018) ${ }^{1}$. Alcohol consumption is the world's third largest risk factor for several disease and disability in the world today. Since India has the second largest population in the world the magnitude of the problem of "Alcoholism" is also very

\section{Corresponding author: \\ Chinnu Sebastian}

Ph.D Scholar, Department of social work, Amrita Vishwa Vidyapeetham, Coimbatore, India; Email Id: chinnumarysebastian2@gmail.com, Mobile Number: +91 9496055243. high in India, and as per WHO 33\% of India's population is alcoholics. It is also seen an annual rise in the number of people who use alcohol (WHO, 2010) ${ }^{2}$. Alcoholism is a major cause of mental, social, family and health problems in Kerala. The state of Kerala has the highest per capita alcohol consumption in India, at over 8 litres per person per year ${ }^{3}$.

Alcohol addiction can be considered as a family problem and this contributes to the increased stress in the family. Family failure and breakage due to alcoholism is a serious pervasive social issue. Alcohol is connected with domestic violence, low satisfaction in the relations, relationship conflicts, under care of children, legal and economic issues, disrupted family interaction etc. and some also results in physical and psychological illness ${ }^{4}$. Researcher observed that the 
actual sufferers are the members of family who depends upon the alcoholic, particularly the spouse. The wives of alcoholics often shows many mental and physical problems, their social involvement will be very low and low level of marital satisfaction. Even these people have very bad communication level also ${ }^{5}$. The spouses of alcoholics have psychological issues in various adaptive and maladaptive coping mechanisms, to restore the equilibrium and to relieve stress.

Family is the basic and fundamental part in the society. And several families contribute to form a society. The health of a nation can be measured only in terms of health of a family. Addiction affects mostly the spouse in the family as the family income is spent on liquor and the spouse is compelled to manage family finance and needs. There exist fear and uncertainty which leads to lack of self- esteem and self- confidence. Spouses of alcohol dependent person may have feelings of guilt, shame, anger, fear, grief, avoidance of social contacts and isolation due to the presence of an alcohol dependent in the family. They are often subjected to moderate to severe form of harassment. Conflict and tense atmosphere arises when they confront the drinking behaviour of their alcohol abusing family member. The problems that arise as a result of alcohol abuse is numerous, which includes long absences from home, destroying house hold and domestic items, no communication, isolation, domestic violence etc. these people are least bothered of the happenings in their family.

Alcoholism of husband results in the change in the interaction patterns of the wife and causes an alteration the complexion of family environment by financial resources of the family which intern results in the dysfunction of several other areas. The heavy stress experienced by wife in dealing with the pressure of her husband will always influence their marital life negatively. Almost all of the alcoholic dependents and their spouses agree that the main factor of marital happiness is related to family interaction and interpersonal relationship ${ }^{6}$.

Family interaction patterns are defined as those socio-psychological transactions occurring in the family as system to evolve processes for decision making, emotional expression, personal views, assigning tasks and social status, enabling the family members to contribute to the growth of the family by generating morphogenesis at emotional, intellectual, and social levels by manipulation of external and internal milieu of the family as a whole. From the evolutionary point of view, every family has patterns of leadership, communication, role, reinforcement, cohesiveness and social support constituting its functioning.

Reinforcement: process adopted by the family to enable the members to imbibe socially approved behaviour. Social support system: manipulation of internal and external social milieu of the family for its existence and growth. Role: socio culturally prescribed and ascribed tasks to be performed by different family members according to their age and sex. Communication: Communication within the family is extremely important because it enables members to express their needs, wants, and concerns to each other. Cohesiveness: processes adopted by the family for a firm degree of mutual trust and interpersonal commitment. Leadership: A family member engaged in decision making through consensus for growth of the family, as a system is the leader of the family ${ }^{7}$.

The researcher visited various De-addiction centres in Kottayam district, Kerala and interacted with the spouse of alcoholics during the $\mathrm{Al}$-anon programme (Al-anon group is a worldwide fellowship that offers a programme of recovery for the families and friends of alcoholics, whether or not the alcoholic recognizes the existence of a drinking problem or seeks help $)^{8}$ and family counselling. The interaction with spouses and discussion with family counsellors of the de-addiction centres helped the researcher to find out the family interaction pattern of the respondents. Researcher came to know that the impact of alcoholism on marital family functioning and influences the family interaction.

\section{Methodology}

This paper is based on standardized scale and the data was collected with an interview schedule from wives of alcoholic attending family counselling and Al-anon meeting in a De-addiction centre of Kottayam district of Kerala. An interview schedule was used to collect data. Family interaction Pattern Scale (FIPS) developed by Bhatti et al (1986) is used for the study. The research is descriptive in nature. Census method used to collect 
the samples. The researcher collected data's from all the respondents who were willing to participate in the study during the period of data collection (July2019 - December 2019). The criterion for selection of the respondents was the wives living with the alcoholics who are under treatment at de-addiction centres in Kottayam District. 200 respondents were included in the study.

\section{Major Findings:}

The simple percentage of socio demographic data reveals the following information. It is seen that $30 \%$ of the respondents are in the 20-30 years age group. Nearly half of the respondents (49\%) are Christian. More than half of the respondents (55\%) are from rural area. More than half of the respondents (56\%) are from nuclear family. Nearly half of the respondents (29\%) have Under Graduate education. Nearly half of the respondents (29\%) are unemployed spouses (housewives). Majority of the respondents $(64 \%)$ have family monthly income above Rs.20000. Half of the respondents (50\%) have above 10 years of drinking habit in spouses. Level of Family interaction pattern

Nearly half of the respondents (37\%) have medium level of Family interaction pattern. 33\% have high family interaction pattern and $30 \%$ have low level of family interaction pattern.

Evidences show that severe alcoholism leads to marital problems and family conflict, and negative emotions and interpersonal interactions often lead to relapses among alcoholics. Stress from family and other relations will motivate them for more drinking. Family members of the alcoholic have medium level of communication as either of the spouses, children or parents is afraid to talk to each other. This is mainly due to the violent nature of the alcoholic where the family members limit their communication so as to avoid problems.

\section{Factors affecting the Family interaction pattern}

Table No. 1: ANOVA Test to compare Family Interaction Pattern (FIP) and Socio demographic profile

\begin{tabular}{|l|l|l|l|l|}
\hline S.No. & Variables & F -value & Table value & Significant/Not \\
\hline 1 & Age & 4.230 & 3.883 & $* *$ \\
\hline 2 & Religion & 0.196 & 3.048 & NS \\
\hline 3 & Area of residence & 4.052 & 3.048 & $*$ \\
\hline 4 & Type of family & 0.946 & 3.042 & NS \\
\hline 5 & Education & 3.482 & 3.113 & NS \\
\hline 6 & Occupation & 0.662 & 2.418 & NS \\
\hline 7 & Family monthly income & 0.915 & 3.042 & $* *$ \\
\hline 8 & Duration of habit in spouse & 4.144 & 3.883 & $*$ \\
\hline
\end{tabular}

NS-Not significant, * -Significant at 5\% level, ** -Significant at $1 \%$ level

1) FIP Score has significant difference with age. The post hoc test indicates that respondents are between the age group of 31-40 years have better FIP.

This study revealed that the family interaction pattern is more significant in spouses of alcoholics with age groups of 31-40 years than other age group.
It is because couple's given more concentration to their young children and when the child grown to teenage or above they leaves the home for studies, job.

2) FIP Score has significant difference with Area of residence. The post hoc test indicates that respondents who are from rural area of residence have better FIP. 
The National Family Health Survey 2015-16(NFHS4) reveals that the consumption of alcohol is higher in rural area than urban area in Kerala ${ }^{9}$. The study revealed that the FIP is significant in rural area than urban and sub urban areas. The significance is due to the influence of social support and interaction among the neighbours is higher in rural area than urban and sub urban areas. The women in the rural areas are gathering together once in a week for the "Kudumbashree Meeting" (a poverty eradication mission by Government of Kerala) which help them to share their problems with the peer group and find the solutions for the same.

There is a need of detailed study among the rural populations, mainly family centered people in order to have in-depth knowledge. On their particular needs and to contribute more to adopt public health measures for preventing these issues and promoting health and psychological rehabilitation. The problem of excessive alcohol consumption is a major cause of public health concern both in urban and rural areas.

3) FIP Score has significant difference with education. The post hoc test indicates that respondents were educated up to 0-9 standard (below $10^{\text {th }}$ standard) has better FIP.

The FIP is higher in respondents below $10^{\text {th }}$ standard education than the educated wives. As most of the respondents are from rural area, the culture of the society is more family oriented. The less educated respondents are more flexible to adjust with alcoholic habit of their spouses than the educated respondents. Hence the FIP is higher in this category. Some of the high income individuals are not highly educated. They are into business and earn lakhs of rupees and hence were found to lead a comfortable life with the satisfaction given by money ${ }^{10}$.

4) FIP Score has significant difference with Duration of habit in spouses. The post hoc test indicates that respondents whose husband's are drinking above 10 years have better FIP.

Analysis of the research shows that the FIP is higher in wives of alcoholics whose spouse's drinks alcohol for more than 10 years. On interaction with the respondents the researcher identified that most of the respondent's spouses who drinks alcohol for more than 10years have more responsibility towards the family and spouses gives mental support to the alcoholics as they were adjusted with habit of the spouses. This helped them to reduce domestic violence and quarrels among them.

\section{Discussion and Conclusion}

Family education and family therapy will lead to solve the problem to a certain extent and other important is to educate the community. Adjustmental problem of wives of alcoholics affect their future generation. Mass media- information should be given about the ill effect of alcoholism. Family counselling or 12 steps therapy (Al-anon) will help the wives to recover from adjustmental problem, marital dissatisfaction and family interaction. De-addiction centres suggest a need to study the stress perceived by spouses of alcoholics and these centres recognized spouse of alcoholic's as an important components in the development of personality, marital conflict, maintenance relationship and treatment of psychological problems. Changes in the attitude and behaviour of the wives of alcoholics can be bought through various therapies like individual, family, musical, occupational etc. and also methods like yoga, meditation and relaxation techniques may contribute to their psychological improvement.

\section{Conclusion}

Nearly half of the respondents (37\%) have medium level of Family interaction pattern. The study reveals that FIP Score has significant difference with age, area of residence, education and duration of drinking habit in spouses. The post hoc test indicates that respondents between the age group of 31-40 years, rural area of residence, below $10^{\text {th }}$ standard education and above 10 years of drinking habit in spouses have better FIP.

Researcher observed that the social consequences of the wives of alcoholics at the individual level significantly impact on personal life, work related area and family relationship. They experience physical, social, economical and psychological problems like socially isolated, lost status in the society, anxiety, depression, loneliness, worthlessness, fear of being rejected etc. because of their husband's drinking. So many negative social consequences like very low selfesteem, disturbed family, social problems, recreational problems etc. are reported from the wives of alcohol 
dependents. It is the responsibility of social workers to use social work intervention techniques and methods (psychotherapy for couples \& family members, group therapy, counselling etc.) for improving their self esteem and better family interaction pattern.

Marital satisfaction and stability is negatively affected because of excessive drinking.

Discordant alcohol consumption is a prime factor in marital dissatisfaction.

Treatment for the couples for alcoholism has given better outcome and greater satisfaction in marital life. This has also resulted in less partner violence.

- After family therapy, the openness, warmth, support and relationships became more effective and sharing of responsibilities is seen in the family.

Practising problem-solving skills and additional behavioural skills help the couples to cope up with relapse episodes ${ }^{11}$.

Ethical Clearance- Taken from Human Ethics Committee, Dept. Of Social work, Amrita Vishwa Vidyapeetham, Coimbatore, Tamil Nadu, India.

Source of Funding- Self

\section{Conflict of Interest- Nil}

\section{References}

1. World Health Organization, (2018). The global burden of disease: Department of mental health\& substance abuse, WHO, Geneva.

2. Gururaj, G, Girish, N \& Benegal, V, et al. Burden and socioeconomic impact of alcohol, the Bangalore study, World Health Organization, South East Regional office, New Delhi.

3. Poonam Binayak. What you need to know about Kerala's love affair with alcohol. Culture trip. 8
July 2017. [https:// the culturetrip.com/asia/india/ articles].

4. Senthil, M \& Manisha Kiran. Family interaction pattern among caregivers of patients with epilepsy and alcohol dependence. IOSR Journal of humanities and social science (IOSR-JHSS). Sept 2015; 20(9): 01-09.

5. Senthil, M. Family interaction pattern and codependency in spouses of alcohol dependence in comparison with normal control. International Journal of Research Granthaalayam.Feb 2016; $4(2)$.

6. Prescila Sharon, S. Perceived quality of life among wives of alcoholics and wives of non-alcoholicsA comparative study. Indian journal of applied research (Social science). Dec 2014; 4(12).

7. Ranbir S Bhatti, Subba Krishna, D K \& Benedicta L Ageira. Validation of family interaction patterns scale. Indian journal of psychiatry. July 1986; 28(3): 211-216.

8. Al-anon, Wikipedia. [https:// en.wikipedia.org]. Al-anon family group headquarters, Inc. Retrieved 2014-01-17.

9. Outlook Web Bureau. Folks, Kerala women are warning up to booze and men ditching it. 05April 2018. [https: //www.outlookindia.com/website/ story].

10. Rajeev, A, Sherin B Abraham \& Thushara G Reddy, et al. A community study of alcohol consumption in a rural area of south India. Int J Community Med Public Health. Jun 2017; 4(6): 2172-2177.

11. Kenneth E Leonard \& Rina D Eiden. Marital and family processes in the context of alcohol use and alcohol disorders. Annu Rev Cli Psychol. 2007; 3:285-310. 